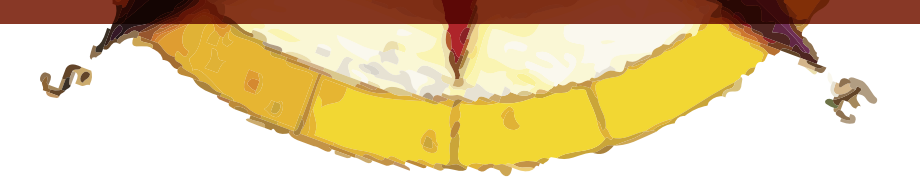

\title{
GEOGRAFÍA, PROBLEMAS SOCIALES Y CONOCIMIENTO ESCOLAR
}

\author{
Francisco F. García Pérez ${ }^{1}$
}

\section{RESUMEN}

La Geografía que se enseña habitualmente en la escuela no suele preparar a los alumnos para comprender y afrontar los graves problemas sociales y ambientales de nuestro mundo. Ello puede observarse, entre otros, en el tipo de conocimiento escolar presente en los libros de texto. Pero el mero cambio del conocimiento escolar de dichos libros no sería una solución suficiente; es necesario un verdadero cambio de la cultura escolar. En ese sentido, trabajar en torno a problemas sociales relevantes, con una perspectiva de investigación escolar, es una alternativa que puede contribuir a la transformación de la educación escolar y, al mismo tiempo, al desarrollo profesional de los profesores.

Palabras clave: Geografía escolar; conocimiento escolar; libros de texto; cultura escolar; metodología de trabajo en torno a problemas; investigación escolar.

\section{RESUMO}

A Geografia que é ensinada na escola geralmente não costuma preparar os alunos para compreender e resolver os graves problemas sociais e ambientais de nosso mundo. Isto pode ser observado, entre outros, no tipo de conhecimento escolar presentes no livros didáticos. No entanto, a simples mudança do conhecimento escolar desses livros não seria uma solução suficiente, precisamos de uma verdadeira mudança na cultura escolar. Nesse sentido, trabalhar em torno de questões sociais relevantes, na perspectiva da investigação escolar, é uma alternativa que pode contribuir para a transformação da educação escolar e ao mesmo tempo, para o desenvolvimento profissional dos professores.

Palavras-chave: Ensino de Geografia; conhecimento escolar; livros didáticos; cultura escolar; metodologia de trabalho em torno de problemas; investigação escolar.

\begin{abstract}
Geography usually taught at school frequently does not prepare students to understand and confront the serious social and environmental problems of our world. It can be observed, among others, in the type of school knowledge in the text books. But the simple change of knowledge in these
\end{abstract}

1 Universidad de Sevilla. 
books would not be a sufficient solution; a real change of school culture is necessary. In this sense, to work on relevant problems, with a perspective of school research, is an alternative that can contribute to the transformation of school education and, at the same time, to the teachers' professional development.

Keywords: School Geography; School Knowledge; Text Books; School Culture; Problem-Based Methodology; School Research

La Geografía ha tenido presencia, de forma continuada, en el currículum escolar desde los orígenes de la escuela en el mundo contemporáneo, habiéndosele reconocido siempre su valor educativo. La Geografía escolar de finales del siglo XIX y de principios del siglo XX tenía una función formativa para los jóvenes estudiantes de ese momento histórico, futuros ciudadanos de una patria, cuyo territorio y actividades humanas se consideraba indispensable conocer. Pero el alumno que se forma hoy como ciudadano del siglo XXI no es ya el alumno del siglo XIX o de principios del siglo XX. Este alumno o alumna no tiene las mismas experiencias ni las mismas expectativas, no se enfrenta a los mismos problemas, no es, simplemente, un ciudadano de su estado-nación, sino, en gran parte, un ciudadano del mundo. Y, si mantenemos el propósito de que la Geografía eduque hoy para ser ciudadanos del mundo (García Pérez y De Alba Fernández, 2008; García Perez, 2011; Souto González, 2011), habría que introducir cambios profundos en su enseñanza para intentar que prepare a esos jóvenes ciudadanos para afrontar los problemas del mundo actual.

\section{¿EDUCA LA GEOGRAFÍA ESCOLAR PARA AFRONTAR LOS PROBLEMAS DEL MUNDO?}

Podemos partir de una serie de preguntas que vayan a la raíz de la cuestión que nos ocupa: ¿está sirviendo la enseñanza de la Geografía para formar ciudadanos y ciudadanas del siglo XXI?, ¿educa la Geografía escolar para entender mejor el cambio climático, el sometimiento de la producción agrícola a los intereses comerciales de las multinacionales, el control de las materias primas básicas por parte de las grandes potencias, el nuevo papel de la economía financiera con respecto a la economía productiva, la insostenibilidad de un modelo de desarrollo basado en el crecimiento continuado, etc., etc.?, ¿prepara la Geografía para analizar los fenómenos en distintas escalas, atendiendo a la interacción entre dichas escalas?... Sin pretender dar una respuesta taxativa y generalizada ${ }^{2}$, podemos afirmar que no. La Geografía enseñada en el sistema escolar no suele ser un conocimiento adaptado al estudio de estos nuevos, graves y urgentes problemas de la humanidad. Los problemas sociales y ambientales de nuestro mundo (un mundo globalizado, informacional, marcadamente urbano) tienen una escala, unas causas, unas consecuencias y, en definitiva, unas características bastante diferentes de los problemas de hace cuatro o cinco décadas; y sin embargo el conocimiento geográfico que se maneja en el sistema escolar (y la forma de manejarlo) sigue siendo sensiblemente igual al de hace décadas; es, por tanto, un conocimiento inadecuado para ayudar a comprender las realidades del siglo XXI y para poder actuar en ellas.

2 Quiero advertir, desde el comienzo de este artículo, que el breve diagnóstico de la situación de la enseñanza de la Geografía y las reflexiones que de ello derivo se basan, fundamentalmente, en la situación mayoritaria de la educación escolar en España, si bien muchos datos indican que la situación es similar en otros países iberoamericanos. En todo caso, hay que conceder no sólo el beneficio de la duda sino todo el apoyo que podamos dar a aquellas iniciativas innovadoras que, desde hace mucho tiempo, han intentado hacer de la enseñanza de la Geografía un instrumento útil para comprender el mundo y transformarlo. En esa tarea estamos, solidarios. 
Este desajuste podemos analizarlo en los diversos planos en que entra en juego dicho conocimiento. En primer lugar, en los libros de texto y materiales didácticos convencionales, como "depósitos" tradicionales de ese saber. En términos generales, creo que se puede afirmar que los contenidos de los libros de texto habituales no abordan los problemas relevantes de nuestro mundo, sino que reproducen un tipo de conocimiento escolar fragmentado, frío, ajeno a los problemas sociales y ambientales reales. La tradición escolar ha ido modelando esos contenidos de la Geografía según un determinado formato, que, por una parte, los hace funcionales para el contexto, restringido, de la enseñanza escolar -de ahí, entre otras razones, su permanencia-, pero, por otra, los ha ido despojando de la potencialidad analítica y explicativa propia del conocimiento científico geográfico. Destacaré brevemente cuatro rasgos caracterizadores de los contenidos de los libros de texto: la superficialidad y dispersión, la codificación del discurso, el carácter de saber diferido y, como colofón, la desvinculación de la realidad.

La presentación de los contenidos -y hablamos de los libros actuales-, más allá de la apariencia de variedad y actualidad informativas, en realidad trivializa y dispersa -en lugar de interconectar- la diversidad de informaciones que se ofrecen en cada tema o lección. En efecto, están presentes en los libros varios tipos de informaciones ${ }^{3}$, pero lo están a modo de "discursos" paralelos (que apenas se entrecruzan o interaccionan); en efecto, poca relación guardan entre sí -dentro de un tema o lección- el discurso textual básico (que es objeto de explicación y de examen), el discurso de las imágenes, el discurso de las informaciones complementarias insertas en recuadros o el discurso de las actividades finales de ampliación y profundización. Todo ello podría ser muy aprovechable, si se estableciera un hilo conductor entre esos diversos materiales, que parece que se hayan ido incorporando al libro a modo de capas sedimentarias. Y, de hecho, así ha sido: como los libros habían de tener información actual, se han añadido actualizaciones sobre el tema, pero sin incidir en el primitivo discurso escolar (que forma una especie de roca base, resistente y duradera); como había que tener en cuenta las concepciones de los alumnos, se ha introducido un ejercicio de exploración inicial (más bien un "examen inicial"), pero que en la práctica no tiene incidencia en la actividad real de la clase, pues, una vez realizado (o ignorado), el docente vuelve al discurso básico del texto; como había que asumir planteamientos de enseñanza activa, se ha añadido al final de la lección un apartado llamado "Investiga sobre...", cuya lógica (y posibilidades de ser realizado en los tiempos escolares) resulta absolutamente extraña a la lógica de explicación/dudas/examen característica del contenido nuclear del libro; como estamos en un mundo icónico, es impensable que un libro actual no contenga buenas imágenes, pero esas imágenes raramente se relacionan o se integran con el discurso central. En definitiva, la roca base apenas resulta afectada por los sedimentos posteriormente depositados; sin duda, harían falta agentes erosivos más potentes.

Además de superficial y dispersa, la información de los libros de texto se presenta codificada según una lógica propia. Asumiendo que el conocimiento geográfico escolar-como el conocimiento de otras materias escolares- tiene unas peculiaridades y una lógica que lo hacen diferente del

3 Hablo conscientemente de "informaciones" y no de "conceptos", porque, pese a que frecuentemente se reprocha a los libros de texto prestar atención casi exclusiva a la dimensión conceptual del conocimiento (en detrimento de las dimensiones procedimental, actitudinal, axiológica), realmente los libros de texto no ofrecen, en la mayoría de las ocasiones, un gran bagaje conceptual, un buen instrumental teórico, sino una gran cantidad de informaciones, datos y hechos, que, perteneciendo, lógicamente, al mundo de lo conceptual, podríamos considerarlas como de un elemental nivel conceptual. ¡Ojalá - podríamos decir- los libros fueran realmente conceptuales!, como ¡ojalá las clases tradicionales, expositivas, verbalistas, fueran realmente "clases magistrales"!, según equívocamente se las llama a veces. 
conocimiento científico de referencia ${ }^{4}$, no es difícil constatar que los contenidos escolares presentes en los libros de texto aparecen "empaquetados" y "codificados" de una manera muy diferente de cómo se genera y difunde el conocimiento científico; una manera que, sin embargo, nos resulta "natural" (porque es lo que estamos acostumbrados a ver desde siempre en el contexto escolar), pero que no facilita un aprendizaje verdaderamente significativo de los alumnos y alumnas. Así, podemos observar un "discurso" que se estructura en epígrafes o apartados y, dentro de cada uno, en una breve introducción, definiciones, enumeración de características, ejercicios para comprobar que se ha memorizado lo escrito-explicado, etc. Desaparece, así, cualquier atisbo de planteamiento de interrogantes, de dudas, de debates, y, por supuesto, al presentar unos contenidos cerrados y acabados, se ignora el carácter de construcción del conocimiento, característica básica de cualquier conocimiento científico.

Como tercer rasgo, podemos señalar que, al presentarse como general y con validez para cualquier uso, el conocimiento de los libros de texto se ofrece como un bagaje cultural útil para ser usado en diversas situaciones y contextos, pero realmente no hay conexiones con problemas concretos ni adaptaciones a situaciones y contextos diversos; se parte del supuesto de que la formación básica proporcionada por ese acervo cultural valdrá al estudiante, futuro ciudadano, para comprender y actuar en relación a las situaciones vitales que se encontrará más adelante. Se ofrece, pues, en la escuela, un conocimiento con una función "diferida", para ser usado en un futuro, indefinido, no para utilizarlo aquí y ahora. Huelga señalar que en esas hipotéticas situaciones futuras raramente los ciudadanos -antiguos estudiantes- hacen uso de ese presunto bagaje formativo, entre otras razones porque -usando un símil del mundo de la informática- no encuentran la ruta para localizar esos antiguos archivos, probablemente deteriorados o borrados.

Y, por todo lo dicho, el conocimiento geográfico escolar -el más habitual; salvemos algunas excelentes excepciones- resulta ajeno, desvinculado de los problemas sociales y ambientales reales, como si, en último término, la validez de ese conocimiento estuviera garantizada en la "burbuja escolar", pero perdiera sus propiedades cuando entra en contacto con el mundo exterior. De esta manera, por una parte, se constata -después de un siglo y medio de historia escolar- la segregación entre el conocimiento escolar y el conocimiento científico de referencia y, por otra, se nos hace presente, también, la desvinculación del conocimiento escolar con respecto al mundo del conocimiento cotidiano. Entender las relaciones entre estos tres tipos de conocimiento sigue siendo una tarea básica de la didáctica.

Sobre la desvinculación, o ignorancia, de los libros de texto con respecto a los graves problemas del mundo -asunto central para enfocar la educación de los ciudadanos y ciudadanas del siglo XXI, como empecé señalando al comienzo- se podrían aportar muchos ejemplos demostrativos. Voy a citar simplemente los resultados de una investigación llevada a cabo por la Comisión de Educación

4 Las peculiaridades del conocimiento escolar con respecto al conocimiento científico de referencia ha sido un aspecto resaltado ya en muchas publicaciones (véase, por ejemplo, recientemente: Cavalcanti, 2008; Callai, 2011). Una mirada muy productiva sobre esta cuestión nos la proporciona, sobre todo, la línea de investigación de historia de las disciplinas escolares (por ejemplo, Goodson, 1995), teniendo un especial interés el enfoque "genealógico". En ese sentido, simplemente a modo de ilustraciones paradigmáticas, se podrían citar la línea clásica de investigación sobre la Geografía escolar desarrollada bajo la coordinación de H. Capel (Capel et al., 1983; CapeL, 1989; Melcón, 1995), el análisis sociogenético de la idea de "estudio del entorno" (Romero y Luis, 2008; MateoS, 2011) o el estudio, en un campo paralelo, del "código disciplinar de la Historia", realizado por R. Cuesta Fernández (1997). 
Ecológica de Ecologistas en Acción (2000) sobre el "currículum oculto antiecológico" de los libros de texto. En dicho estudio, realizado sobre una amplia muestra de libros, se llegaba a la conclusión de que los contenidos de los mismos, en general, eran ajenos a los graves problemas ambientales y sociales del mundo. No aparecen, en efecto, en la mayoría de los libros analizados, los conceptos de sostenibilidad e insostenibilidad, ocultándose así la gravedad de la crisis que afecta al modelo de desarrollo dominante en nuestro mundo y manteniendo, explícita o implícitamente, una perspectiva de progreso indefinido de la humanidad. Por lo demás, se aprecia en los libros una exaltación incondicional de la tecnología (y en especial del transporte a larga distancia y a alta velocidad) como, supuesta, solución de los problemas de la humanidad; la naturaleza, la tierra y la vida se muestran subordinadas a la economía y al mercado; la historia sigue siendo, básicamente, una historia de los estados y del poder; las actividades de la mitad de la humanidad, las mujeres, casi no son tomadas en consideración; se ocultan o apenas se tratan asuntos como: el poder de las multinacionales y de los grandes grupos creadores de opinión, el control de la agricultura a través de la industria de semillas y fertilizantes, el agotamiento de los combustibles fósiles, la desaparición de muchas culturas consideradas "atrasadas", el papel de los movimientos alternativos, etc.

Constatado que el conocimiento presente en los libros de texto tiene estas características, el problema no se solucionaría, meramente, elaborando otros materiales alternativos, pues sabemos como diversos estudios han venido mostrando ${ }^{5}$ - que el conocimiento escolar sufre procesos sucesivos de reconstrucción: en la formulación del currículum, en los libros de texto y materiales escolares -como acabo de exponer-, pero también en el discurso que se desarrolla en el aula, mediante la interacción profesor-alumnos... ¿cuál es el conocimiento que queda, como resultado del aprendizaje, en los alumnos tras estas reconstrucciones?, ¿en qué se parece lo que queda al primitivo conocimiento de la Geografía escolar?, ¿en qué se parece lo que queda al conocimiento científico geográfico de referencia?...

Con frecuencia se ha intentado buscar la renovación del conocimiento escolar en la actualización de los paradigmas científicos de referencia. Pero, siendo importante, tampoco es esa la clave, pues, en último término, aunque se cambien los contenidos de enseñanza, el mecanismo escolar en su conjunto (espacios, tiempos, actores, reglas, etc.) sigue funcionando ${ }^{6}$. En definitiva, la cultura escolar tiende a fagocitar y a remodelar estos intentos renovadores, reformateando esas nuevas aportaciones, como conocimiento escolar convencional. El problema es, pues, más complejo, y ello nos lleva a analizar esta cuestión en términos de choque o, al menos, de desajuste "cultural": la cultura académica de la escuela tiene poco que ver con la cultura cotidiana del alumnado en esta sociedad de la información; y el problema es que el sistema escolar está mostrando escasa capacidad para superar esa brecha entre culturas (García Díaz et al., 2007; véase también Libâneo, 2006).

Sin duda, hay que generar otro conocimiento escolar, otro currículum que nos permita trabajar, en los distintos niveles escolares, problemas verdaderamente relevantes, a fin de que los ciudadanos que se están formando en el sistema escolar se preparen mejor para afrontar los retos actuales y futuros de nuestro mundo. Y ello hay que concebirlo como un auténtico "cambio cultural" radical. Esto nos introduce -como insinuaba más arriba- en el análisis de las complejas relaciones del co-

$5 \quad$ Puede consultarse, al respecto, por ejemplo, Merchán, 2011.

6 La permanencia de estos mecanismos del sistema escolar ha sido explicada con diferentes conceptos. Destaco el concepto de "gramática escolar" de D. Tyack y L. Cuban (2001) o el de "cultura escolar", utilizado por A. Viñao (2002). 
nocimiento escolar con el conocimiento científico y con el conocimiento cotidiano, un asunto que habría que abordar, asimismo, con detenimiento ${ }^{7}$.

\section{TRABAJAR EN TORNO A PROBLEMAS COMO ALTERNATIVA}

Si buscamos una alternativa que nos permita enseñar Geografía en la escuela para formar a un alumnado capaz de afrontar los problemas sociales y ambientales de nuestro mundo, la opción que se nos presenta como más coherente -aun sabiendo que habría que transformar, a la vez, otros aspectos de la estructura escolar- es, justamente, trabajar en la escuela sobre esos problemas.

La idea, general, de "trabajar sobre problemas o en torno a problemas" es una opción educativa con hondas raíces en las corrientes pedagógicas renovadoras y se suele vincular a la idea de "investigación escolar". Seguirle la pista a la idea nos llevaría lejos; en un rápido recordatorio, podríamos citar como fuentes de legítima inspiración los social studies norteamericanos (destaquemos a J. Dewey), la "Escuela Nueva", las "técnicas" de C. Freinet, los "temas generadores" de la pedagogía de P. Freire, algunos proyectos curriculares clásicos, como el Humanities Curriculum Project, de L. Stenhouse, etc., etc.

Hay un fundamento básico para proponer esta forma de trabajo: el proceso de abordar diariamente situaciones novedosas (problemáticas, en definitiva) contribuye a que los seres humanos, desde que somos niños, vayamos construyendo nuevo conocimiento (entendido en un amplio sentido), de forma que se puede decir que aprendemos en la medida en que trabajamos con esas situaciones problemáticas y elaboramos respuestas (cognitivas, afectivas, conductuales) adecuadas a las mismas. En cierta manera, pues, en la vida diaria constantemente "investigamos". Así entendida, la investigación sería como una estrategia básica de conocimiento y actuación en la realidad, propia del comportamiento de los seres humanos y con un claro valor adaptativo tanto para el individuo como para la propia especie (García Díaz y García Pérez, 2000). Bien es verdad -como señalaré más adelante- que el contexto escolar presenta peculiaridades que no presentan otros contextos sociales, sobre todo por su carácter intencional en relación con la educación; pero, al fin y al cabo, no deja de ser un contexto en el que interactúan personas y grupos con intereses diversos desarrollando determinadas actividades, por lo que las posibilidades de sentir curiosidad por algo, de mostrar interés por lo que se hace, de colaborar en una tarea de búsqueda, etc., en principio, están ahí presentes, al menos como posibilidad que puede ser desarrollada mediante una intervención didáctica pertinente.

E1 trabajo en torno a problemas ha sido asumido como una alternativa en varias áreas de conocimiento, especialmente en la didáctica de las matemáticas, de las ciencias naturales y de las ciencias sociales. También ha sido seña de identidad de muchos colectivos y grupos innovadores que desarrollan proyectos alternativos. Así, por ejemplo, en el Proyecto IRES ("Investigación y Renovación Escolar" $)^{8}$ o en las propuestas didácticas de los grupos vinculados a la Federación Icaria ${ }^{9}$ Por lo

$7 \quad$ A esta cuestión se dedica, por ejemplo, el n ${ }^{\circ} 75$ (2011) de la revista Investigación en la Escuela.

8 Sobre el Proyecto IRES en general, puede consultarse García Pérez y Porlán, 2000; para entender el marco de las propuestas del IRES puede consultarse, asimismo, García Díaz, 1998 y García Pérez, 2000; el profesorado -entre el que me cuento- que trabaja en el contexto del IRES constituye la Red IRES, cuya página web puede consultarse en http://www.redires.net/. 
demás, el trabajo en torno a problemas relevantes ha sido objeto de interesantes revisiones y debates didácticos ${ }^{10}$. Este enfoque, asimismo, ha estado presente en la Geografía escolar, no sólo por su preocupación tradicional por el estudio del entorno ${ }^{11}$, sino, sobre todo, por la incorporación - no todo lo frecuente que hubiera sido deseable- de los planteamientos de los geógrafos sociales y, más concretamente, de los geógrafos críticos.

Esta rápida revisión de las raíces de la opción de trabajar en torno a problemas nos lleva, inevitablemente, a plantearnos la idea, más general, de un currículum concebido como "integrado" y no como una suma de disciplinas ${ }^{12}$. Aunque no podamos abordar, en este momento, la cuestión del currículum integrado -que nos llevaría, por lo demás, al análisis de otros planteamientos próximos como los enfoques interdisciplinares y transversales-, sí es conveniente dejar constancia de dicho marco, pues la propia idea de problema, tal como la estoy presentando, tiende a superar los límites de los campos disciplinares, para partir de la realidad social (que no está dividida en disciplinas), como inicio de la investigación, y retornar a ella, con propuestas de acción transformadora. Lo dicho no invalida, en todo caso, la posibilidad de plantear y trabajar los problemas desde el ámbito de una materia escolar, como es el caso de la Geografía, siempre, claro está, que adoptemos como marco una Geografía abierta a la conexión con los problemas sociales y ambientales reales.

En definitiva, estamos hablando de trabajar, desde la Geografía -y, en general, desde las Ciencias Sociales- sobre "problemas sociales y ambientales" -"socioambientales", los denominamos en el proyecto IRES, para destacar la estrecha conexión de lo ambiental con lo social- que sean realmente relevantes. Pero ¿qué significa eso desde una perspectiva didáctica? Podríamos preguntarnos a ese respecto: ¿se trataría de trabajar, directamente, los problemas reales de nuestra sociedad?; ¿serían los problemas considerados como tales por la cultura dominante?; ¿serían, por el contrario, los problemas identificados y denunciados desde posiciones sociales críticas?; ¿se trataría de los problemas estudiados por la Geografía o por otras ciencias sociales?; ¿coincidirían, en todo caso, dichos problemas con aquellos asuntos que los alumnos se plantean como problemas en su mundo cotidiano?...

Podemos comprobar que esos "problemas relevantes" son entendidos de diferente manera dependiendo de la perspectiva y del contexto en que se plantean. Así, el conocimiento cotidiano, muy marcado por los medios de comunicación -en definitiva por la cultura hegemónica-, tiende a contemplar estos problemas de una determinada forma: con una escala de análisis muy reducida, tanto desde el punto de vista espacial como temporal, y con una perspectiva simplificadora de los mismos; en definitiva una visión propia de la cultura de la superficialidad que el pensamiento dominante

9 En relación con la Federación Icaria, o Fedicaria, un colectivo -en el que, asimismo, estoy integrado- que trabaja por una didáctica crítica de las Ciencias Sociales, puede consultarse la web correspondiente: http://www.fedicaria.org/.

10 Véase, a este respecto, por ejemplo: Souto González, 1998; Luis Gómez, 2001; Fernández Martínez et al., 2006; Ollé, 2011.

11 Las investigación sobre la historia o, mejor, sobre la genealogía de las materias escolares está arrojando luz sobre la formación y sobre la presencia de determinados contenidos en la cultura escolar, como anteriormente he señalado. Me refiero ahora, más concretamente, a las aportaciones relativas a la genealogía del "estudio del entorno", trabajada por autores -antes citados- como J. Mateos (2011), que acuña el concepto de "código pedagógico del entorno", o por J. Romero y A. Luis (2008), quienes realizan un interesante análisis crítico de la trayectoria de ese contenido escolar.

Sobre la opción de un currículum integrado sigue siendo útil la consulta de la obra, ya clásica, de Beane, 2005. 
-que se presenta como "pensamiento único"- impone. Y en ese contexto cultural se socializan las nuevas generaciones (García Díaz, 2004; García Pérez y De Alba Fernéndez, 2008).

Los alumnos, por consiguiente, suelen estar impregnados de esta visión, dadas sus vivencias habituales, si bien no hay que olvidar que el "conocimiento de los alumnos" presenta, a su vez, un carácter peculiar -bastante "mestizo", podríamos decir-, pues ellos también han ido incorporando a su visión del mundo interpretaciones característicamente "escolares" (que entran en interacción con las ideas que les impregnan en los ámbitos cotidianos no escolares).

Por otra parte, la Geografía, y, en general, las Ciencias Sociales -o, al menos, determinados paradigmas o enfoques de dichas disciplinas- aportan un análisis de esos problemas de una gran complejidad y potencialidad transformadora, lo que permite, sin duda, análisis de la realidad mucho más profundos de los que se suelen hacer en el ámbito cotidiano. Para el caso concreto de la Geografía, se han acuñado expresiones como "pensar el espacio", para destacar el carácter de reflexión problematizada de las realidades espaciales (superando la mera descripción) propia del conocimiento geográfico. Asimismo, el concepto de "espacio social", manejado por Milton Santos y otros geógrafos críticos, incorpora la idea de "problematización del espacio"13.

Sin embargo no se puede olvidar que el planteamiento de las disciplinas científicas acerca de estos problemas se realiza en un contexto, con unos propósitos, con un método de trabajo, con unos resultados distintos de aquellos que se hallan en la lógica de los alumnos y en el conocimiento cotidiano (García Díaz, 2001). Por tanto, no basta con estar de acuerdo en suscitar o plantear este tipo de problemas en la enseñanza, no bastaría tampoco con abordarlos desde la posición de la Geografía o de otras disciplinas sociales, sino que habría que tener un mínimo de garantías acerca de que los alumnos pudieran realmente llegar a aprender lo que se les propone. En ese sentido, cuando desde la didáctica se plantea la conveniencia de utilizar "situaciones-problema", se destaca la necesidad de que esas situaciones estén adaptadas a los procesos de aprendizaje del alumno, es decir, que el problema pueda ser reconocido como tal por el sujeto que aprende; más concretamente, se trataría de proponer un problema a la vez suficientemente próximo al alumno para que pueda abordar su tratamiento y suficientemente alejado para que resulte enigmático y atractivo y le obligue, por tanto, a dar pasos en busca de soluciones.

En definitiva, es necesario tener en cuenta no sólo la relevancia social del problema sino también su carácter de problema planteado en el contexto escolar, ese contexto escolar que desnaturaliza los conocimientos que en él entran, reconstruyéndolos -como se decía más arriba- como un nuevo y peculiar conocimiento, con "formato escolar"; y esto obliga a adoptar una perspectiva más compleja, una perspectiva desde la didáctica. En el proyecto IRES -al que me he referido anteriormente- se defiende un currículum organizado en torno a "problemas socioambientales relevantes", en correspondencia con una concepción del conocimiento escolar deseable concebido como "enriquecimiento del conocimiento de los alumnos", desde las formulaciones más elementales, marcadas fuertemente por el conocimiento cotidiano, hacia formulaciones progresivamente más complejas, sin que la meta tenga que ser, obligadamente, "el conocimiento científico". Y esta opción se justifica desde diversas perspectivas (García Pérez y Porlán, 2000; García Pérez, 2000): desde una perspectiva sistémica y compleja, los problemas constituyen un planteamiento más abierto (y lleno de posibilidades;

13 Sobre esta cuestión y, en general, sobre la concepción del espacio en la Geografía puede consultarse, lógicamente, una extensa bibliografía. Cito, simplemente, como referencias: Unwin, 1995 y Souto González, 1998. 
por tanto más complejo) del conocimiento; desde una perspectiva constructivista, trabajar en torno a problemas es el planteamiento coherente con los supuestos de interrogarse acerca del conocimiento y de irlo construyendo en la interacción; desde una perspectiva social crítica, son los problemas sociales (y ambientales) los que deben ser objeto de enseñanza y no el conocimiento como producto "valioso" legado por las tradiciones disciplinares -conocimiento que, en todo caso, tendría que estar al servicio del tratamiento de los problemas, y no a la inversa-.

Los problemas, en esta formulación didáctica que estoy propugnando, deberían ofrecer la posibilidad de ser trabajados en diversas escalas, tanto espaciales como temporales. En efecto, al señalar la necesidad de que los problemas sociales y ambientales que trabajemos se presenten adaptados al contexto de nuestros alumnos, no queremos decir que hayan de ser forzosamente abordados como problemas a escala local para después ir ampliando la escala de análisis. Renunciando a la lógica de "círculos concéntricos" 14 , se trataría más bien de asumir "una lógica de progresión desde planteamientos más sencillos a planteamientos más complejos; $y$, en ese sentido, puede haber problemas que, siendo lejanos en el espacio, puedan sin embargo resultar próximos al alumnado y, además, puedan ser susceptibles de formulaciones más simples" (García Pérez y De Alba Fernández, 2008).

Asimismo, hay que advertir que nos estamos refiriendo a los problemas no en su mera dimensión sincrónica, sino con una perspectiva diacrónica o, mejor, genealógica, es decir, que la enseñanza, "al interrogarse sobre los problemas del presente, indaga, sin fronteras temporales preconcebidas, sobre la génesis de su constitución en tanto que problemas relevantes de los seres humanos"; de esta forma, se recurre a "lo histórico" como "una demanda necesaria no de la propia disciplina, sino de los problemas que se abordan y de la perspectiva crítico-dialéctica ensayada", lo que, por tanto, "no determina a priori la escala temporal más conveniente" para utilizar en el tratamiento del problema (Cuesta Fernández, p. 83). Porque, en realidad, lo que nos interesa educativamente es "el presente", que no habría que confundir con "lo contemporáneo". En este sentido, habría que intentar abordar los problemas según la secuencia didáctica presente->pasado->presente->futuro, otorgando un papel central al presente (como punto de partida y de llegada), recurriendo al pasado (como búsqueda de la explicación histórico-genética) y contemplando el futuro (como referencia para la actuación, frente al olvido de que suele ser objeto en la lógica escolar).

Manejar esta relevancia múltiple en la formulación y tratamiento didáctico de los problemas, en el contexto escolar, no es, desde luego, tarea fácil. De ahí la importancia, en definitiva, de la profesora o del profesor, como experto en estos procesos. Es él quien tendría que combinar adecuadamente, desde su cosmovisión educativa, esos tres criterios básicos: la relevancia del problema desde el punto de vista social y ambiental, la posibilidad de ser trabajado por el alumnado y el tipo de aportaciones científicas que pueden hacer más provechoso el trabajo sobre ese problema (García Pérez y De Alba Fernández, 2008).

\section{TRABAJAR LOS PROBLEMAS CON UNA METODOLOGÍA DE INVESTIGACIÓN ESCOLAR}

Lo dicho anteriormente nos lleva a la cuestión del método de trabajo o metodología didáctica. Y la metodología adecuada para trabajar problemas es, lógicamente, una metodología de "investi-

14 Una lógica suficientemente criticada desde la didáctica de la Geografía (véase, por ejemplo: Souto González, 1998; Romero y Luis, 2008). 
gación". Esta metodología trata de generar situaciones en el aula que permitan que los problemas objeto de estudio sean asumidos y trabajados como tales por el alumnado implicado. Para ello las actividades didácticas tienen que seleccionarse, organizarse y secuenciarse de forma que propicien el surgimiento y planteamiento de los problemas, su tratamiento mediante el manejo de nuevas informaciones adecuadas y la elaboración, progresiva, de conclusiones en torno a lo trabajado. Lo esencial no es, pues, tanto definir unas fases o pasos metodológicos cuanto entender la "investigación escolar" como un proceso de construcción conjunta del conocimiento, en el que tanto el profesor como los alumnos comparten tareas, pero tienen papeles y responsabilidades diferentes. En todo caso, hay cierto consenso en señalar como pautas básicas de esta metodología estas cuatro, que hemos asumido en el proyecto IRES (García Pérez, 2000):

1. Partir del planteamiento y delimitación de uno o de varios problemas. Se trataría, ante todo, de que el proceso de enseñanza- aprendizaje parta de que los alumnos y alumnas lleguen a asumir las cuestiones que hayan de trabajar como verdaderos "problemas", es decir como algo que les interese realmente, que estimule en ellos actitudes de curiosidad (en un sentido amplio) y que tenga potencialidad para desencadenar un proceso que desemboque, en definitiva, en la construcción de nuevos conocimientos y en el desarrollo de conductas adecuadas. El que los alumnos asuman una cuestión como problema no implica forzosamente que ellos mismos sean quienes la planteen y la formulen; puede hacerlo el profesor, consiguiendo que los alumnos lo acojan posteriormente. Lo que es realmente más importante es que el problema se mantenga asumido como tal a lo largo de todo el proceso de trabajo, con los cambios y reformulaciones que pueda ir sufriendo.

2. Contar con las concepciones de los alumnos sobre esos problemas y ponerlas en juego a lo largo del proceso. Si asumimos, como referencia de partida, que los aprendizajes escolares se producen por interacción entre las ideas, concepciones o representaciones de que disponen los alumnos y las nuevas informaciones que se introducen en el proceso de enseñanza, no podemos considerar como "erróneas" las ideas de nuestros alumnos sobre los problemas trabajados (por el hecho de que no se ajusten al análisis que se podría hacer de dichos problemas desde una perspectiva científica), sino como bases o puntos de engarce sobre los que se irán construyendo los nuevos conocimientos. Y esto implica tomar en consideración las concepciones o representaciones de los alumnos (es decir, su modelos interpretativos del mundo, su lógica de razonamiento, los estereotipos sociales asumidos procedentes del pensamiento dominante...) no simplemente al principio del proceso de trabajo sino durante todo el desarrollo del mismo.

3. Trabajar con nuevas informaciones en relación con los problemas que se estén tratando. Frente a las posiciones de los modelos basados en el "descubrimiento espontáneo", hay que destacar la importancia de esta pauta metodológica, es decir, la necesidad de incorporar al proceso de enseñanza -y, por tanto, al proceso de aprendizaje- informaciones realmente "nuevas" (en relación con las que habitualmente se manejan en los contextos cotidianos, no escolares) y de suficiente potencialidad para poder comprender en profundidad los problemas trabajados y poder intervenir, en su momento, más adecuadamente en relación con los mismos. Por ello, hay que poner en juego un conocimiento escolar enriquecido, de calidad, que, de manera intencional, hay que introducir, de formas pautada, en el proceso para hacer evolucionar las concepciones más simples (y condicionadas fuertemente por el conocimiento cotidiano) de los alumnos. Desde esta perspectiva el conocimiento científico puede jugar aquí un importante papel, muy distinto del que convencionalmente juega. 
4. Elaborar conclusiones sobre los problemas trabajados y definir posibles líneas de actuación. En un modelo metodológico de estas características las conclusiones se van obteniendo, en un proceso continuado, al mismo tiempo que se van construyendo nuevos conocimientos y generando, en consecuencia, nuevos tipos de comportamiento en relación con los problemas trabajados, en un proceso de interacción entre las concepciones primeras que tenían los alumnos y las informaciones nuevas aportadas en el proceso. En todo caso, es muy pertinente plantear, específicamente, tareas de síntesis de los resultados de los problemas trabajados, lo que contribuiría a fijar lo aprendido. Y quizás la forma más adecuada para consolidar lo aprendido y vincularlo, realmente, a la intervención e integración en la realidad es proporcionar la posibilidad de poner en práctica los nuevos aprendizajes, de forma que se pueda comprobar el interés y la utilidad de los mismos en la acción, también en contextos sociales fuera de la escuela.

Con la sintética justificación realizada en el apartado anterior y con la breve descripción de estas pautas básicas, pretendo simplemente caracterizar el modelo metodológico de trabajo en torno a problemas relevantes, consciente, en cualquier caso, de que se han propuesto y experimentado una gran diversidad de métodos de enseñanza que, de una u otra forma, se consideran como "metodología de investigación"15. Conviene, en todo caso, no confundir la idea de "investigación escolar" con otras propuestas de "enseñanza activa", que podríamos considerar más bien como de "descubrimiento autónomo" o de "descubrimiento espontáneo", propuestas éstas centradas en la actividad del alumno, con tareas muy abiertas y poco programadas, que pretenden imitar, de alguna manera, el proceso de la investigación científica de referencia, intentando hacer del alumno un "pequeño geógrafo" o un "pequeño historiador". Tampoco habría que confundirla con la modalidad de "investigación guiada", que busca parecidos objetivos, pero bajo el control del profesor, que impone, en este caso, su lógica de construcción del conocimiento.

En todo caso, conviene no olvidar que trabajar en torno a problemas no es sólo una cuestión de método de trabajo, sino también, y en primer lugar, una cuestión de contenido, o, más exactamente, de formulación del contenido, seleccionado conforme a los criterios de relevancia citados más arriba y organizado y secuenciado según niveles progresivos de complejidad, de manera que un determinado problema, que se planteara inicialmente como próximo a la experiencia del alumno iría evolucionando, diversificándose y complejizándose, en el propio proceso de su tratamiento.

\section{DIFICULTADES Y POSIBILIDADES DEL TRABAJO EN TORNO A PROBLEMAS}

El interés educativo de organizar los contenidos de enseñanza en torno a problemas relevantes parece fuera de duda. Pero trabajar esos problemas para que los alumnos puedan comprenderlos y aprender lo que nos proponemos enseñarles no constituye una tarea fácil, en el contexto real del aula, ya que esta opción innovadora no se acomoda bien a la lógica de la cultura escolar, en la que se suele admitir como "natural" que hay unos contenidos que ya vienen dados por la propia tradición escolar, que hay unos profesores cuyo rol sería el de transmitir ese legado y que hay unos alumnos (y sus familias) que parecen esperar que se les transmita, por los procedimientos conocidos, el conocimiento acabado que, posteriormente habrán de "devolver" a su profesores mediante el mecanismo habitual de evaluación, los exámenes. 
Efectivamente, los profesores y profesoras mostramos una comprensible tendencia al uso de planteamientos metodológicos que faciliten una compacta exposición de los contenidos previstos y, en todo caso, una rápida realización de las actividades correspondientes, sin que resulte, en principio, fácil y apetecible seguir unas pautas metodológicas más centradas en los procesos de aprendizaje del alumno por investigación. También los alumnos y alumnas suelen tener dificultades para asimilar y madurar estrategias y técnicas de trabajo propias de una metodología basada en el trabajo sobre problemas, por resultar ajena a las pautas que suelen ser más habituales en su educación académica y por la carencia de confianza en su propia capacidad para plantearse problemas y trabajar con ellos. La organización escolar y el contexto social, por su parte, favorecen poco, por diversos motivos (horario, rutinas escolares, presión de los padres respecto a lo que creen que deben hacer los niños en la escuela...) la aplicación de esta metodología (García Pérez y Rivero, 1995).

Así, pues, la incorporación a las experiencias escolares de un modelo de de enseñanza basado en el tratamiento de problemas no es tarea fácil, dada la tradicional resistencia del sistema escolar a la introducción de innovaciones que pudieran, a la larga, poner en peligro su estructura básica. De ahí la existencia de esas dificultades y bloqueos que acabo de señalar. Pese a todo, considero que hay que ratificarse en la necesidad de definir mejor y experimentar adecuadamente este modelo que, como hipótesis de partida, considero con mayores potencialidades que otros cuyas limitaciones -por suerte o por desgracia- conocemos.

Por lo demás, este modelo de trabajo vinculado a la "idea de investigación" es aplicable-desde la perspectiva del Proyecto IRES- tanto al trabajo del alumno -tal como acabo de exponer- como al trabajo del profesor, considerado, en ese sentido, como verdadero "profesor investigador en el aula", una opción profesional que tiene, igualmente, profundas raíces en la tradición innovadora de la profesionalidad docente.

En efecto, como antes he señalado, el profesorado es un factor clave a la hora de desarrollar el trabajo en torno a problemas; y sería muy difícil que un profesor o profesora desarrolle de forma coherente ese tipo de trabajo docente si no tiene asumida, como referente profesional, esa misma filosofía, es decir, si no está convencido de que él mismo también aprende y se desarrolla profesionalmente "investigando" ${ }^{16}$. Y ello nos lleva inevitablemente a postular un modelo de formación del profesorado alternativo a los tradicionales (Romero Morante et al., 2006), un modelo de desarrollo profesional, en definitiva, que propicie otro tipo de relación del profesorado con el conocimiento y suponga un mayor compromiso profesional con la función, social y comprometida, de enseñar. De ahí que, para nosotros en el Proyecto IRES, trabajar a favor de un modelo alternativo de enseñanza y trabajar en la construcción de un modelo alternativo de docente sean dos tareas estrechamente vinculadas.

En el proyecto IRES venimos trabajando en la experimentación de propuestas curriculares estructuradas en torno al trabajo sobre problemas sociales y ambientales relevantes. En el marco del IRES se ha concretado, por ejemplo, el proyecto Investigando Nuestro Mundo 6-12, planteado como experimentación, con seguimiento investigador, de un currículum alternativo del área de Conocimiento del Medio en Primaria, y estructurado, de manera integrada, en base a diversos "ámbitos de investigación escolar" (Cañal, Pozuelos y Travé, 2005). En el IRES los "ámbitos de investigación

16 Véase más ampliamente desarrollada nuestra posición al respecto, por ejemplo, en: Porlán y Rivero, 1998 y García Pérez, 2006. 
escolar" se conciben como conjuntos de problemas sociales y ambientales, relacionados entre sí y que son relevantes para la comprensión de la realidad desde la perspectiva de los alumnos, al tiempo que permiten relacionar e integrar conjuntos de contenidos desde la lógica del conocimiento escolar deseable (García Pérez, 2000). Así, por ejemplo, pueden funcionar como ámbitos de investigación escolar temáticas como la alimentación, el hábitat o las actividades económicas.

Desde estos supuestos y desarrollando investigación didáctica sobre la experimentación curricular de propuestas de trabajo en torno a problemas, la Geografía puede contribuir decisivamente -pese a las dificultades del marco escolar- a educar a los ciudadanos y ciudadanas - no solo futuros, sino ya actuales, aunque sean jóvenes- para ser capaces de afrontar los graves problemas de nuestro mundo en el siglo XXI.

\section{BIBLIOGRAFÍA}

Beane, J. A. (2005). La integración del currículum. El diseño del núcleo de la educación democrática. Madrid: Morata.

Callai, H.C. (2011). A geografia escolar - e os conteúdos da geografía. Anekumene, 1(1), 128-139. $<$ http://www.anekumene.com/index.php/revista/article/view/14> consultado el 06/11/11.

Cañal, P. (2007). La investigación escolar, hoy. Alambique. Didáctica de las Ciencias Experimentales, (52), 9-19.

Cañal, P; Pozuelos, F.J. y Travé, G. (2005). Proyecto Curricular Investigando Nuestro Mundo. Descripción General y Fundamentos. Sevilla: Díada.

Capel, H. (1989). Historia de la ciencia e historia de las disciplinas científicas. Objetivos y bifurcaciones de un programa de investigación sobre historia de la geografía. Geocrítica, (84).

Capel, H. et al. (1983). Ciencia para la burguesía. Renovación pedagógica y enseñanza de la geografía en la revolución liberal española (1814-1857). Barcelona: Publicaciones de la Universidad de Barcelona.

Cavalcanti, L. de S. (2008). Concepções de Geografia e de Geografia escolar no mundo contemporâneo. In Cavalcanti, L. de S. A Geografia Escolar e a Cidade: ensaios sobre o ensino de Geografia para a vida urbana cotidiana. Campinas-SP: Editora Papirus, 15-37.

Cuesta Fernández, R. (1997). Sociogénesis de una disciplina escolar: la Historia. Barcelona: PomaresCorredor.

Cuesta Fernández, R. (1999). La educación histórica del deseo. La didáctica de la crítica y el futuro del viaje a Fedicaria. Con-Ciencia Social, (3), 70-97.

Ecologistas en Acción: Comisión de Educación Ecológica (2000). Estudio del currículum oculto antiecológico de los libros de texto. (Versión reducida; 185 p.). Disponible en: <http://www.ecologistasenaccion.org/IMG/pdf/Informe_curriculum.pdf> consultado el 10/11/11.

Fernández Martínez, M.; García Sánchez, J.N.; De Caso, A.; Fidalgo, R. y Arias, O. (2006). E1 
aprendizaje basado en problemas: revisión de estudios empíricos internacionales. Revista de Educación, (341), 397-418. <http://www.revistaeducacion.mec.es/re341_17.htm> consultado el 28/10/11.

García Díaz, J.E. (1998). Hacia una teoría alternativa sobre los contenidos escolares. Sevilla: Díada.

García Díaz, J.E. (2001). De los problemas científicos a los problemas socioambientales (y vuelta). Alambique. Didáctica de las Ciencias Experimentales, (29), 25-33.

García Díaz, J.E. (2004). Educación Ambiental, Constructivismo y Complejidad. Sevilla: Díada Editora.

García Díaz, J.E. y García Pérez, F.F. (2000). Aprender investigando. Una propuesta metodológica basada en la investigación. Sevilla: Díada Editora, $6^{\mathrm{a}}$ ed. (1 $1^{\mathrm{a}}$ ed. 1989).

García Díaz, J.E.; García Pérez, F.F.; Martín, J. y Porlán, R. (2007). ¿Son incompatibles la escuela y las nuevas pautas culturales? Investigación en la Escuela, (63), 17-28.

García Pérez, F.F. (2000). Un modelo didáctico alternativo para transformar la educación: el Modelo de Investigación en la Escuela. Scripta Nova, Revista Electrónica de Geografia y Ciencias Sociales. Barcelona: Universidad de Barcelona, 4(64). $<$ http://www.ub.es/geocrit/sn-64.htm $>$ consultado el 28/10/11

García Pérez, F.F. (2006). Formación del profesorado y realidades educativas: una perspectiva centrada en los problemas prácticos profesionales. In Escudero, J.M. y Luis, A. (eds.). La formación del profesorado y la mejora de la educación. Políticas y prácticas. Barcelona: Octaedro, 269-309.

García Pérez, F.F. (2011): Problemas del mundo y educación escolar: un desafío para la enseñanza de la geografía y las ciencias sociales. Revista Brasileira de Educação em Geografia, 1(1), 108-122.

<http://www.revistaedugeo.com.br/ojs/index.php/revistaedugeo/article/view/18/16> consultado el 10/10/11.

García Pérez, F.F. y De Alba Fernández, N. (2008). ¿Puede la escuela del siglo XXI educar a los ciudadanos y ciudadanas del siglo XXI? Scripta Nova. Revista Electrónica de Geografia y Ciencias Sociales. Barcelona: Universidad de Barcelona, 12 (270), 122. <http://www.ub.es/geocrit/ sn/sn-270/sn-270-122.htm> consultado el 10/10/11.

García Pérez, F.F. y Porlán, R. (2000). El Proyecto IRES (Investigación y Renovación Escolar). Biblio 3W. Revista Bibliográfica de Geografia y Ciencias Sociales. Barcelona: Universidad de Barcelona, 5 (205). <http://www.ub.es/geocrit/b3w-205.htm> consultado el 28/10/11.

García Pérez, F.F. y Rivero, A. (1995). Dificultades y obstáculos en la construcción del conocimiento escolar en una hipótesis de progresión de lo simple a lo complejo. Reflexiones desde el ámbito del medio urbano. Investigación en la Escuela, (27), 83-94.

Goodson, I.F. (1995). Historia del Currículum. La construcción social de las disciplinas escolares. Barcelona: Pomares-Corredor. 
Libâneo, J.C. (2006). Cultura jovem, mídias e escola: o que muda no trabalho dos profesores? Educativa, 9(1), 25-46. <http://seer.ucg.br/index.php/educativa/article/view/73> consultado el 10 de octubre de 2011.

Luis Gómez, A. (2001). Tradiciones curriculares, innovaciones educativas y función social conservadora del conocimiento escolar: la primacía de los temas sobre los problemas. Biblio $3 \mathrm{~W}$. Revista Bibliográfica de Geografia y Ciencias Sociales. Barcelona: Universidad de Barcelona, 30 de diciembre de 2001, vol. VI, $\mathrm{n}^{\circ} 337$. < http://www.ub.es/geocrit/b3w-337.htm > consultado el 8 de octubre de 2011.

Mateos, J. (2011). Genealogía de un saber escolar: el código pedagógico del entorno. Barcelona: Octaedro.

Melcón, J. (1995). La renovación de la enseñanza de la geografía en los orígenes de la España contemporánea. Cuadernos del ICE, Madrid: Ediciones de la Universidad Autónoma de Madrid (14).

Merchán, F.J. (2011). Práctica de la enseñanza y gobierno de la clase. Revista española de pedagogía, (250), 521-535.

Ollé, M. (2011). Ensenyar geografia a partir de situacions problema. Perspectiva Escolar, (358), 14-23.

Porlán, R. y Rivero, A. (1998). El conocimiento de los profesores. Una propuesta formativa en el área de ciencias. Sevilla: Díada.

Pozuelos, F.J. (2001). La investigación escolar: Una alternativa para innovar en el aula. In Pozuelos, F.J. y Travé, G. (eds.). Entre pupitres. Razones e instrumentos para un nuevo marco educativo. Huelva: Publicaciones de la Universidad de Huelva, 113-151.

Romero Morante, J. y Luis Gómez, A. (2008). El conocimiento socio-geográfico en la escuela: las tensiones inherentes a la transmisión institucionalizada de cultura y los dilemas de la educación para la democracia en este mundo globalizado. Scripta Nova. Revista Electrónica de Geografia y Ciencias Sociales. Barcelona: Universidad de Barcelona, 12 (270), 123. <http://www. ub.es/geocrit/sn/sn-270/sn-270-123.htm> consultado el 10 de octubre de 2011.

Romero, J.; Luis, A.; García Pérez, F.F. y Rozada, J.Mª (2006): La formación del profesorado y la construcción social de la docencia. Con-Ciencia Social, (10), 15-68.

Souto González, X.M. (1998). Didáctica de la Geografia. Problemas sociales y conocimiento del medio. Barcelona: Ediciones del Serbal.

Souto González, X.M. (2011). Una educación geográfica para el siglo XXI: Aprender competencias para ser ciudadano en el mundo global. Anekumene, 1( 1), 28-47. < http://www.anekumene. com/index.php/revista/article/view/8> consultado el 06 de noviembre.

Tyack, D. y Cuban, L. (2001). En busca de la utopía. Un siglo de reformas de las escuelas públicas. México: Fondo de Cultura Económica. 
Unwin, T. (1995). El lugar de la geografia. Madrid: Cátedra.

Viñao, A. (2002). Sistemas educativos, culturas escolares y reformas. Continuidades y cambios. Madrid: Morata.

Artículo recibido 20 - 09 - 11. Aprobado 25 - $11-11$. 